\title{
Constitutive and induced insect resistance in RNAi-mediated ultra-low gossypol cottonseed cotton
}

\author{
Steffen Hagenbucher ${ }^{1}$, Michael Eisenring ${ }^{1}$, Michael Meissle ${ }^{1}$, Keerti S. Rathore ${ }^{2}$ and Jörg Romeis ${ }^{{ }^{*}}$ (D)
}

\begin{abstract}
Background: Besides fibers, cotton plants also produce a large amount of seeds with a high oil and protein content. The use of these seeds is restricted by their high contents of the terpenoid gossypol, which is harmful to humans and livestock. Using a genetic engineering approach, "Ultra-low gossypol cottonseed" (ULGCS) plants were produced by knocking down an enzyme that catalyzes the formation of a precursor of gossypol. This was accomplished via RNAimediated silencing of the target gene using a seed-specific a-globulin promotor. Since gossypol is also a crucial defense mechanism against leaf-feeding herbivores, ULGCS plants might possess lower herbivore resistance than nonengineered plants. Therefore, we tested the constitutive and inducible direct insect resistance of two ULGCS cotton lines against the African cotton leafworm, Spodoptera littoralis.

Result: The herbivore was equally affected by both ULGCS lines and the control (Coker 312) line when feeding on fully expanded true leaves from undamaged plants and plants induced by jasmonic acid. When plants were induced by caterpillar-damage, however, S. littoralis larvae performed better on the ULGCS plants. Terpenoid analyses revealed that the ULGCS lines were equally inducible as the control plants. Levels of terpenoids were always lower in one of the two lines. In the case of cotyledons, caterpillars performed better on ULGCS cotton than on conventional cotton. This was likely caused by reduced levels of gossypol in ULGCS cotyledons.
\end{abstract}

Conclusion: Despite those effects, the insect resistance of ULGSC cotton can be considered as largely intact and the plants may, therefore, be an interesting alternative to conventional cotton varieties.

Keywords: ULGCS, Glandless cotton, Gossypium hirsutum, Gossypol, Spodoptera littoralis, Genetically modified crops, TAM66274

\section{Background}

Cotton plants from the genus Gossypium are one of the major sources of fiber. Today $95 \%$ of all cotton are derived from Gossypium hirsutum (Malvaceae: Malvales) [1]. Besides its fibers, cotton plants also produce a large amount of seeds (1.65 kg seeds per $\mathrm{kg}$ lint) [2]. The seeds are rich in protein and are a valuable source of oil and fodder $[3,4]$. However, they typically contain high concentrations of the terpenoid gossypol, present within the glands of seed kernels. Most of the other aerial parts of cotton plants also have subepidermal glands and those in the green parts of the plant contain not just gossypol,

\footnotetext{
* Correspondence: joerg.romeis@agroscope.admin.ch

${ }^{1}$ Agroscope, Research Division Agroecology and Environment,

Reckenholzstrasse 191, 8046 Zürich, Switzerland

Full list of author information is available at the end of the article
}

but also hemigossypolone and heliocides $1-4$, all derived from the same biosynthetic pathway. Gossypol is known to be toxic to non-ruminant animals [5]. Therefore, a major goal in cotton breeding has been to select for plants that do not produce gossypol. Breeders managed to achieve this goal by producing so-called glandless cotton [2]. However, gossypol and related terpenoids are important herbivore and pathogen resistance factors which are inducible by leaf-chewing herbivores or the direct application of the phytohormone jasmonic acid, a key regulator of cotton defense responses [6]. Glandless cotton is therefore more susceptible to insect pests and diseases [6, 7]. Therefore, there is a need for cotton plants that produce seeds with low levels of gossypol, but unchanged amounts of terpenoids in other plant parts. Using a genetic engineering approach, such

(c) The Author(s). 2019 Open Access This article is distributed under the terms of the Creative Commons Attribution 4.0 International License (http://creativecommons.org/licenses/by/4.0/), which permits unrestricted use, distribution, and 
“Ultra-low gossypol cottonseed" (ULGCS) plants were produced by knocking down the production of $\delta$-cadinene synthase, an enzyme that catalyzes the formation of $\delta$-cadinene, a precursor of gossypol. This was accomplished via RNAi-mediated silencing of the target gene using a seed-specific $\alpha$-globulin promotor $[8,9]$. One transformation event TAM66274 (line 66-274 in our manuscript) has been approved by US regulators in October 2018 [10]).

One concern related to these ULGCS plants is that they could have an increased susceptibility to herbivores and/ or diseases. Previous work on induced terpenoid production has focused on salicylic acid driven resistance against diseases [11], and no data exists on the jasmonic acid driven induction pathways that are commonly associated with damage from foliage and fruit-feeding pests of cotton such as Heliothis, Helicoverpa and Spodoptera species (all Lepidoptera: Noctuidae). As cotton terpenoids are important for cotton insect resistance [6,7], changes in this pathway could have significant effects on plant health and ultimately yield. Although Rathore et al. [11] reported that the constitutive terpenoid production in true leaves is not impaired in ULGCS lines, it is greatly reduced in the cotyledons. Therefore, a lack of gossypol in the cotyledons of ULGCS cotton could make seedlings of these plants more sensitive to herbivore attacks than conventional plants. While a previous study did show that the response of ULGCS cotton against the fungal pathogen Rhizoctonia solani (Cantharellales: Ceratobasidiaceae) is intact [11], no data have yet been collected on insect resistance.

In this study, we tested the strength of constitutive and induced insect resistance in ULGCS cotton using two different transgenic events and their non-transformed parental cv. Coker 312. We tested the performance of the generalist herbivore Spodoptera littoralis (Lepidoptera: Noctuidae) as a model species. The moth is a multivoltine, polyphagous herbivore found in Africa, the Middle East and the Mediterranean area and is known to attack at least 130 different plants species in 56 families [12]. Spodoptera littoralis is considered an important pest species and is mainly a foliage-feeder, compared to other pests in cotton that are fruit-feeders. Additionally, this species is an important model species for plant-insect interactions, which makes it easier to put data into a wider context.

We fed larvae of $S$. littoralis with cotyledons from the three selected cotton lines and recorded several performance parameters in order to test if the low gossypol content of ULGCS cotton could benefit the herbivore. Gossypol concentrations of the cotyledons were measured via a high-performance liquid chromatography (HPLC) to link changes in larval performance to changes in plant chemistry. In a second step, we analyzed the larval performance on the true leaves of the selected cotton lines. Larvae were fed with leaves from plants which were either undamaged, damaged by $S$. littoralis larvae, or induced by treatment with jasmonic acid. Additionally, we assessed the larval behavior under choice conditions to detect potential changes in attraction toward the different plants.

\section{Results \\ Cotyledon assay \\ Terpenoid content}

Cotyledons contained gossypol, but no measurable amounts of hemigossypolone, heliocides $\mathrm{H} 1+\mathrm{H} 4$, and heliocides $\mathrm{H} 2+\mathrm{H} 3$. Gossypol concentrations were much lower in the two ULGCS cotton lines (day $1: \mathrm{F}_{2,78}=83.6$, $p<0.001$; day $4: \mathrm{F}_{2,81}=67.7 ; p<0.001$ ), and in some cases not detectable (RNAi 1: day 1: $28.6 \%$ of tested plants, day 4 : $20.0 \%$ of tested plants; RNAi 2: day 1: $33.3 \%$ of tested plants, day $4: 38.9 \%$ of tested plants) when compared to non-transgenic near isoline (Coker 312) (Fig. 1). Gossypol concentrations in cotyledons from the non-transgenic plants declined significantly between the start of the assay (day 1 ) and the time point when the second cotyledon was harvested (day 4) $\left(\mathrm{F}_{1,62}=9.2, p=0.004\right)$ (Fig. 1).

\section{Larval performance}

When fed with cotyledons from all three cotton lines, no effect was visible on the survival of $S$. littoralis larvae during the seven day feeding period (Table 1). However, differences in larval weight were evident after four and seven days, with weights of the larvae being significantly lower on Coker 312 plants compared to the two ULGCS lines. After seven days, larvae had consumed significantly less material from the Coker 312 leaf discs compared to the two ULGCS lines (Table 1).

\section{True leaf assay}

\section{Terpenoid content}

Hemigossypolone concentration was affected by plant type and treatment but there was no significant interaction detected (Plant: $\mathrm{F}_{2,187}=5.3, p=0.006$; Treatment $\mathrm{F}_{2,185}=49.7, p<0.001$, Plant $\times$ Treatment $\mathrm{F}_{4,181}=1.0$, $p=0.389$ ). Gossypol concentration was affected by plant type and treatment but there was no significant interaction detected (Plant: $\mathrm{F}_{2,183}=12.0, p<0.001$; Treatment $\mathrm{F}_{2}, 181=23.6, p<0.001$, Plant $\times$ Treatment $\mathrm{F}_{4}$, $177=1.0, p=0.390)$. Heliocide $1+4$ concentration was not affected by plant type, but by treatment. There was no significant interaction detected (Plant: $\mathrm{F}_{2,184}=2.67$, $p=0.072$; Treatment $\mathrm{F}_{2,182}=27.92, p<0.001$, Plant $\times$ Treatment $\mathrm{F}_{4,178}=0.85, p=0.498$ ).

The youngest leaves of untreated ULGCS and Coker 312 cotton contained significant amounts of gossypol, hemigossypolone, and heliocides $\mathrm{H} 1+\mathrm{H} 4$. We were not able to verify the presence of the heliocides $\mathrm{H} 2+\mathrm{H} 3$. The concentrations of hemigossypolone and gossypol were equal in Coker 312 and RNAi 2, but significantly 


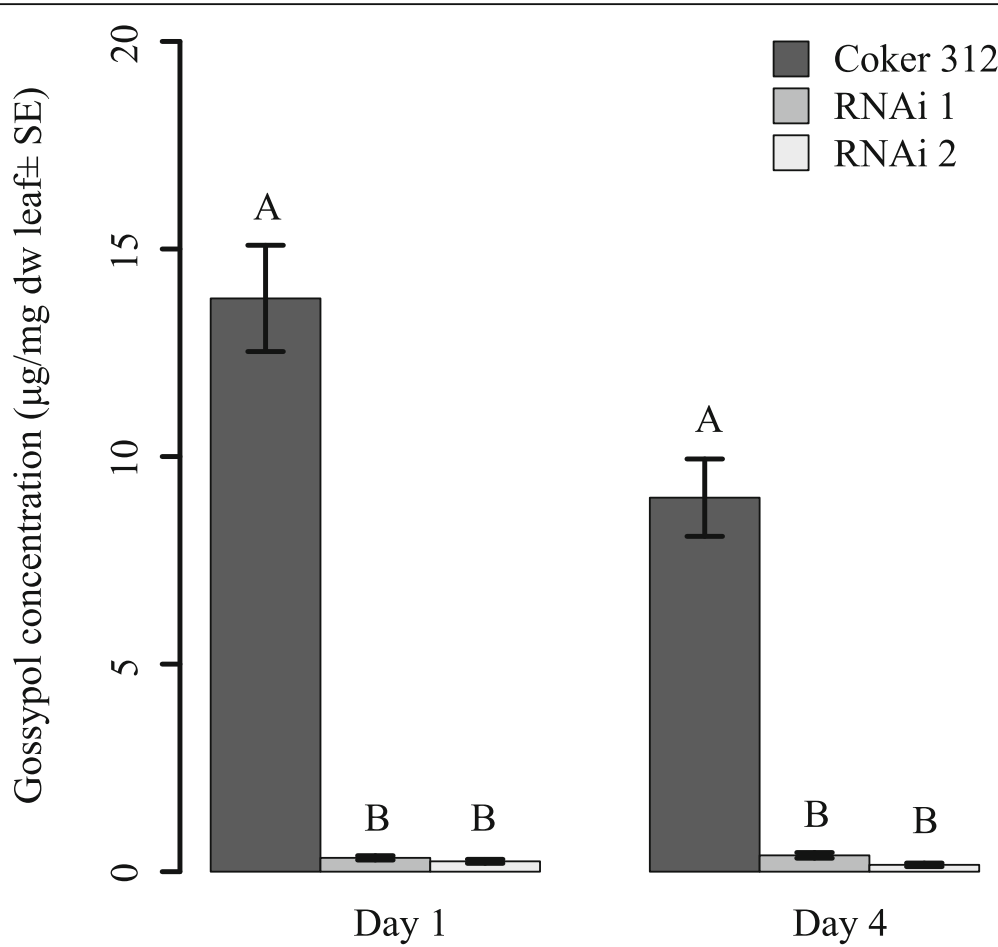

Fig. 1 Concentration of gossypol in cotyledons as affected by plant type. Gossypol levels ( $\mu \mathrm{g} / \mathrm{mg} \pm \mathrm{SE}$ ) in cotyledons from conventional cotton (Coker 312) and two ULGCS cotton lines (RNAi 1: 66-49B and RNAi 2: 66-274). One cotyledon was collected at the start of the feeding assay (day 1). The second cotyledon was collected on day four of the assay. Different capital letters over bars from the same sample day indicate a significant difference between plant types (Tukey HSD test), $n=32-36$

lower in RNAi 1 (hemigossypolone: $\mathrm{F}_{2,66}=4.95, p=$ 0.021; gossypol: $\mathrm{F}_{2,63}=8.09, p<0.001$; Fig. $\left.2 \mathrm{a}\right)$. Concentrations of heliocides $\mathrm{H} 1+\mathrm{H} 4$ did not differ significantly among plant lines $\left(\mathrm{F}_{2,64}=0.902, p=0.411\right)$.

To induce the plants, they were infested with $S$. littoralis larvae before the start of the experiment. The plant type had no effect on the consumed leaf areas (Coker: $21.9 \pm 2.42 \mathrm{~cm}^{2} \pm \mathrm{SE}$; RNAi 1: $19.4 \pm 2.34 \mathrm{~cm}^{2} \pm$ SE; RNAi 2: $22.6 \pm 2.97 \mathrm{~cm}^{2} \pm \mathrm{SE}$; ANOVA: $\mathrm{F}_{2,50}=$ $0.521 ; p=0.597)$. This leaf damage resulted in a strongly increased concentration of terpenoids in the youngest leaf compared to uninduced plants (Fig. 2b).
Again, leaves from RNAi 1 contained a significantly lower concentration of gossypol and hemigossypolone than the remaining plant types (Fig. 2b) (hemigossypolone $\mathrm{F}_{2,59}=3.185, p=0.049$, gossypol $\mathrm{F}_{2,59}=5.306$, $p=0.008$ ), while no difference was detected for heliocides $\mathrm{H} 1+\mathrm{H} 4\left(\mathrm{~F}_{2,58}=1.639, p=0.203\right)$.

Plants that were treated with jasmonic acid had an increased content of terpenoids compared to uninduced plants, but a lower concentration than plants infested with S. littoralis (Fig. 2c). However, no significant difference in content was found among the three plant types for any of the terpenoids analyzed (hemigossypolone:

Table 1 Performance of Spodoptera littoralis larvae on cotyledons from conventional and ULGCS cotton. First instars were fed with cotyledon leaf discs from conventional cotton (Coker 312) and two ULGCS cotton lines (RNAi 1: 66-49B and RNAi 2: 66-274) for seven days. Survival, weight, and consumed leaf area were measured after four and seven days. Means within one column followed by different letters are significantly different (Tukey HSD test) $(n=30-32)$

\begin{tabular}{|c|c|c|c|c|c|c|}
\hline \multirow{2}{*}{$\begin{array}{l}\text { Cotton } \\
\text { type }\end{array}$} & \multicolumn{3}{|l|}{ Four days } & \multicolumn{3}{|l|}{ Seven days } \\
\hline & $\begin{array}{l}\text { Weight } \\
(\mathrm{mg} \pm \mathrm{SE})\end{array}$ & $\begin{array}{l}\text { Leaf area consumed } \\
\left(\mathrm{cm}^{2} \pm \mathrm{SE}\right)\end{array}$ & Survival (\%) & $\begin{array}{l}\text { Weight } \\
(\mathrm{mg} \pm \mathrm{SE})\end{array}$ & $\begin{array}{l}\text { Leaf area consumed } \\
\left(\mathrm{cm}^{2} \pm \mathrm{SE}\right)\end{array}$ & Survival (\%) \\
\hline Coker 312 & $1.8 \pm 0.23 b$ & $0.46 \pm 0.083$ & 59.4 & $28.9 \pm 4.14 b$ & $2.70 \pm 0.35 b$ & 59.4 \\
\hline RNAi 1 & $2.8 \pm 0.32 \mathrm{a}$ & $0.51 \pm 0.064$ & 82.9 & $43.1 \pm 4.35 \mathrm{a}$ & $4.74 \pm 0.41 \mathrm{a}$ & 77.1 \\
\hline RNAi 2 & $3.3 \pm 0.27 a$ & $0.67 \pm 0.079$ & 72.7 & $49.8 \pm 3.97 \mathrm{a}$ & $5.03 \pm 0.36 \mathrm{a}$ & 69.4 \\
\hline GLM & $\begin{array}{l}F_{2,71}=5.9 \\
p=0.004\end{array}$ & $\begin{array}{l}F_{2,69}=1.9 \\
p=0.160\end{array}$ & $\begin{array}{l}x^{2} 2,100=4.6 \\
p=0.100\end{array}$ & $\begin{array}{l}F_{2,68}=5.9 \\
p=0.004\end{array}$ & $\begin{array}{l}F_{2,68}=11.3 \\
p<0.001\end{array}$ & $\begin{array}{l}x^{2} 2,100=2.5 \\
p=0.290\end{array}$ \\
\hline
\end{tabular}




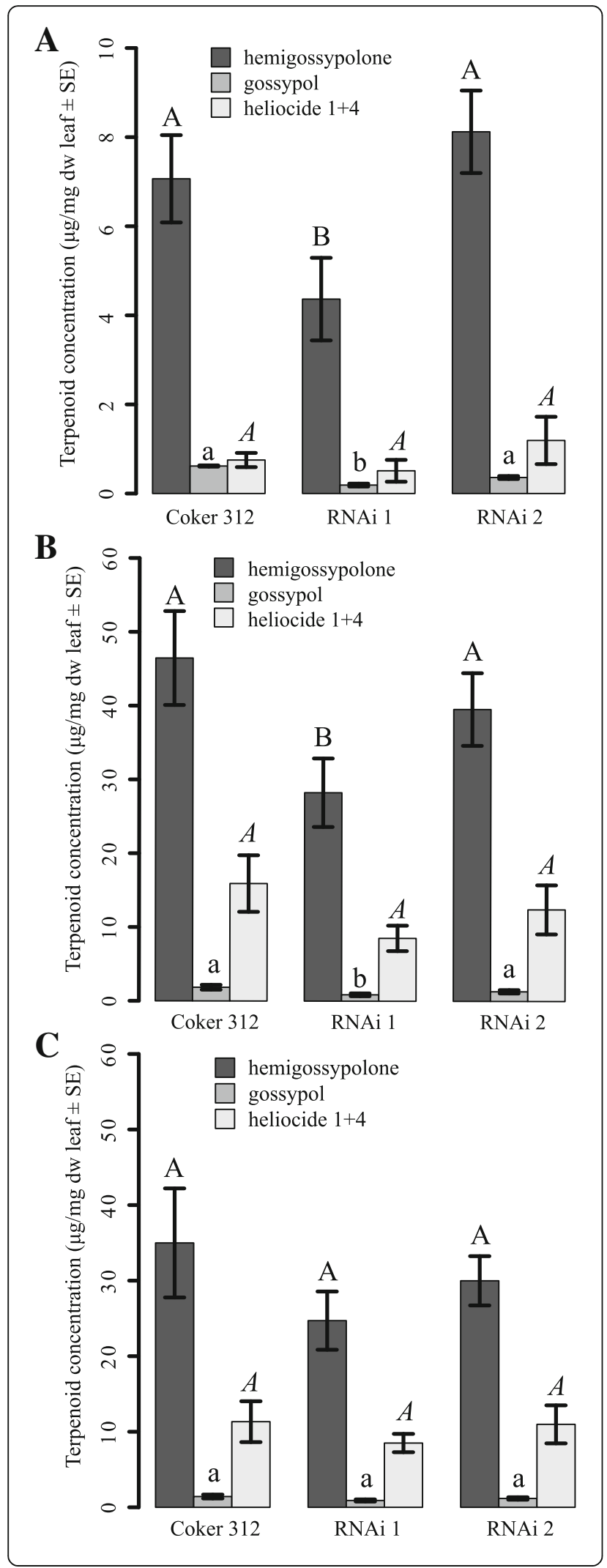

Fig. 2 Impact of plant type and plant induction on concentration of terpenoids in cotton. Terpenoid levels $(\mu \mathrm{g} / \mathrm{mg} \pm \mathrm{SE})$ in the youngest leaves collected from conventional cotton (Coker 312) and two ULGCS cotton lines (RNAi 1: 66-49B and RNAi 2: 66-274). A) Cotton plants were untreated. B) Cotton plants had been exposed to three 2nd instar S. littoralis. C) Cotton plants were treated with jasmonic acid $(4.8 \mu \mathrm{mol})$. Within induction treatments, different letters over the same chemical compound indicate a significantly difference between plant types (upper case letter: hemigossypolone; upper case letter italics: heliocide $\mathrm{H} 1+\mathrm{H} 4$; lower case letter: gossypol) (Tukey HSD test), $n=20-25$

$\mathrm{F}_{2,56}=1.042, p=0.359 ;$ gossypol: $\mathrm{F}_{2,55}=1.838, p=0.169 ;$ $\left.\mathrm{H} 1+\mathrm{H} 4: \mathrm{F}_{2,56}=0.536, p=0.388\right)($ Fig. $2 \mathrm{c}$ ).

\section{Larval performance}

When S. littoralis were feedin on leaf discs from different cotton plants during the first seven days of their development, we found no strong impact of the factor plant type, but a strong difference between induced and uninduced plants. After four and seven days, larvae feeding on leaf discs from untreated plants were generally heavier and had consumed a larger leaf area compared to larvae fed discs from S. littoralis-infested or jasmonic acid-treated plants (Tables 2 and 3). The overall analyses revealed that plant type had no influence on the consumed leaf area and only an influence on larval weight after four days, but not after seven days. However, interactions between plant type and treatment were detectable in all cases. This is best explained by a better performance of larvae on ULGCS cotton lines that had been induced by $S$. littoralis feeding, compared to similarly treated Coker 312, as is evident in an increased larval weight and a larger leaf area consumed. These interactions were assessed with interaction plots, which can be found in the Additional file 1: Figs. S1-S4). Survival was not affected by either plant type or treatment, nor was there a significant interaction (Plant: $\chi^{2}{ }_{2,182}=0.33, p=0.850$; Treatment $\chi^{2}{ }_{2,180}=0.12$, $p=0.940$, Plant $\mathrm{x}$ Treatment $\chi_{2,176}^{2}=0.85, p=0.931$ ).

For a subset of larvae on untreated plants of the three cotton lines, the larvae were allowed to complete their development to the pupal stage. We found no significant differences among plant types for weight after 14 days $\left(\mathrm{F}_{2}\right.$, $47=2.80 ; p=0.07)$, development time $\left(\chi_{2}^{2}, 39=0.65 ; p=\right.$ $0.72)$, pupation rate $\left(X_{2,57}^{2}=0.48 ; p=0.79\right)$, pupal weight $\left(\mathrm{F}_{2,37}=2.36 ; p=0.11\right)$, or sex ratio $\left(\mathrm{X}_{2,39}^{2}=0.67 ; p=0.72\right)$.

\section{Preference assay}

We conducted a series of choice assays to establish if ULGCS cotton types were more attractive for $S$. littoralis larvae than Coker 312 (Table 4). Larvae showed no preference for any of the plant types when the plants were treated equally (i.e., undamaged, damaged by caterpillars, or treated with jasmonic acid). The preference indices 
Table 2 Performance of Spodoptera littoralis larvae on leaves from conventional and ULGCS cotton. First instars were fed with leaf discs from conventional cotton (Coker 312) and two ULGCS cotton lines (RNAi 1: 66-49B and RNAi 2: 66-274) for 7 days. Survival, weight, and consumed leaf area were measured after four and 7 days. Four larvae received leaf material from one plant and were pooled for analyses, 20 plants were tested per treatment $(n=20)$

\begin{tabular}{|c|c|c|c|c|c|c|}
\hline \multirow[t]{2}{*}{ Cotton type } & \multicolumn{3}{|l|}{ Four days } & \multicolumn{3}{|l|}{ Seven days } \\
\hline & $\begin{array}{l}\text { Weight } \\
(\mathrm{mg} \pm \mathrm{SE})\end{array}$ & $\begin{array}{l}\text { Leaf area consumed } \\
\left(\mathrm{cm}^{2} \pm \mathrm{SE}\right)\end{array}$ & $\begin{array}{l}\text { Survival } \\
\text { (Number larvae } \pm \text { SE) }\end{array}$ & $\begin{array}{l}\text { Weight } \\
(\mathrm{mg} \pm \mathrm{SE})\end{array}$ & $\begin{array}{l}\text { Leaf area consumed } \\
\left(\mathrm{cm}^{2} \pm \mathrm{SE}\right)\end{array}$ & $\begin{array}{l}\text { Survival } \\
\text { (Number larvae } \pm \text { SE) }\end{array}$ \\
\hline \multicolumn{7}{|c|}{ Untreated cotton plants } \\
\hline Coker 312 & $3.96 \pm 0.260$ & $0.90 \pm 0.062$ & $3.29 \pm 0.171$ & $36.64 \pm 3.653$ & $5.14 \pm 0.435$ & $3.19 \pm 0.178$ \\
\hline RNAi 1 & $4.14 \pm 0.337$ & $1.12 \pm 0.094$ & $3.27 \pm 0.150$ & $35.78 \pm 3.101$ & $5.33 \pm 0.336$ & $3.14 \pm 0.190$ \\
\hline RNAi 2 & $4.01 \pm 0.218$ & $0.94 \pm 0.056$ & $3.27 \pm 0.199$ & $34.04 \pm 2.832$ & $5.03 \pm 0.267$ & $3.14 \pm 0.190$ \\
\hline \multicolumn{7}{|c|}{ Spodoptera littoralis damaged cotton plants } \\
\hline Coker 312 & $1.29 \pm 0.164$ & $0.40 \pm 0.058$ & $3.40 \pm 0.240$ & $6.38 \pm 0.882$ & $1.18 \pm 0.158$ & $3.15 \pm 0.261$ \\
\hline RNAi 1 & $2.05 \pm 0.239$ & $0.70 \pm 0.081$ & $3.40 \pm 0.152$ & $9.98 \pm 1.726$ & $1.82 \pm 0.308$ & $3.25 \pm 0.160$ \\
\hline RNAi 2 & $1.96 \pm 0.246$ & $0.64 \pm 0.081$ & $3.55 \pm 0.135$ & $12.24 \pm 1.806$ & $1.86 \pm 0.254$ & $3.25 \pm 0.160$ \\
\hline \multicolumn{7}{|c|}{ Jasmonic acid treated cotton plants } \\
\hline Coker 312 & $2.37 \pm 0.159$ & $0.74 \pm 0.058$ & $3.70 \pm 0.128$ & $21.75 \pm 2.585$ & $3.38 \pm 0.302$ & $3.55 \pm 0.135$ \\
\hline RNAi 1 & $2.44 \pm 0.169$ & $0.80 \pm 0.068$ & $3.35 \pm 0.196$ & $19.94 \pm 1.940$ & $3.16 \pm 0.315$ & $3.30 \pm 0.193$ \\
\hline RNAi 2 & $2.72 \pm 0.232$ & $0.78 \pm 0.069$ & $3.05 \pm 0.235$ & $19.92 \pm 2.565$ & $3.22 \pm 0.392$ & $2.95 \pm 0.211$ \\
\hline
\end{tabular}

[calculated as: (leaf area consumed Plant A - leaf area consumed Plant B)/total leaf area consumed] for all of these comparison varied between -0.30 and 0.12 .

Additional assays were performed to assess the response of larvae when given a choice between uninduced and induced (both by $S$. littoralis and jasmonic acid) leaves from the same plant type (Table 4). In these assays, we found a preference for uninduced plant material regardless of plant type or type of induction. When larvae were allowed to choose between undamaged and $S$. littoralis damaged plants, preference indices varied between 0.57 and 0.76 (Table 4). Statistical comparison of the indices showed that this response was similar in the two transgenic lines compared to Coker 312 (Coker vs. RNAi 1: V = 280; $p=0.130$; Coker vs. RNAi 2: V=222; $p=0.665)$. Similar results were obtained in the comparisons between undamaged and jasmonic acid treated plants where the preference indices varied between 0.39 and 0.52 (Table 4). A comparison of the indices revealed no difference between the two transgenic lines and Coker 312 (Coker vs. RNAi 1: V=217; $p=0.665$; Coker vs. RNAi 2 : $\mathrm{V}=175 ; p=0.665)$.

\section{Discussion}

During this study, we assessed whether two ULGCS cotton lines have a reduced insect-resistance compared to conventional, non-transformed cotton plants.

The comparison of terpenoid (hemigossypolone, gossypol, heliocide $\mathrm{H} 1+\mathrm{H} 4)$ concentrations in true leaves from Coker 312 and the two ULGCS lines revealed significantly lower concentrations in the leaves of one of the two ULGCS lines (RNAi 1). The pattern was evident in uninduced as well as in induced plants. This, however, does not seem to be a general side-effect of

Table 3 GLM results of performance assay. Effects of plant type (factor with three levels: Coker 312; RNAi 1 and RNAi 2) and treatment (factor with three levels: untreated, S. littoralis infested and jasmonic acid treated) and their interaction on weight and leaf area consumed by S. littoralis larvae in the performance assay with true leaves after four and seven days $(n=20)$

\begin{tabular}{|c|c|c|c|c|}
\hline & & Plant & Treatment & Plant $\times$ Treatment \\
\hline \multirow[t]{2}{*}{4 days } & Leaf area consumed & $\begin{array}{l}F_{2,181}=1.88 ; \\
p=0.16\end{array}$ & $F_{2,179}=63.3 ; p<0.001$ & $F_{4,175}=3.19 ; p=0.02$ \\
\hline & Weight & $\begin{array}{l}F_{2,181}=4.72 ; \\
p=0.01\end{array}$ & $F_{2,179}=22.3 ; p<0.001$ & $F_{4,175}=2.82 ; p=0.03$ \\
\hline \multirow[t]{2}{*}{7 days } & Leaf area consumed & $\begin{array}{l}F_{2,181}=0.01 \\
p=0.99\end{array}$ & $F_{2,179}=82.3 ; p<0.001$ & $F_{4,175}=3.55 ; p=0.01$ \\
\hline & Weight & $\begin{array}{l}F_{2,181}=0.21 ; \\
p=0.80\end{array}$ & $F_{2,179}=79.4 ; p<0.001$ & $F_{4,175}=2.50 ; p=0.04$ \\
\hline
\end{tabular}


Table 4 Preference of Spodoptora littoralis larvae towards different cotton lines and different treatments. Third instars were given the choice between leaf discs from two different plants (Plant A vs Plant B). The leaf discs were either taken from conventional cotton (Coker 312) or two ULGCS cotton lines (RNAi 1: 66-49B and RNAi 2: 66-274). Additionally, plants were either untreated, damaged by larvae or treated with jasmonic acid. After $24 \mathrm{~h}$ the consumed leaf surface was measured. Preference indices were calculated as: (leaf area consumed Plant A - leaf area consumed Plant B)/total leaf area consumed. Three larvae received leaf discs from one plant pair and data were pooled for analyses, twenty plant pairs were tested per comparison $(n=20)$. Un $=$ untreated; Spod = damage by S. littoralis larvae; JA = treated with jasmonic acid. The consumed leaf areas for Plant A and Plant B were compared using one-sided Wilcoxon signed-rank tests

\begin{tabular}{|c|c|c|c|c|c|}
\hline Plant A & Plant B & Leaf area Plant $\mathrm{A}\left(\mathrm{cm}^{2} \pm \mathrm{SE}\right)$ & Leaf area Plant $\mathrm{B}\left(\mathrm{cm}^{2} \pm \mathrm{SE}\right)$ & Preference for Plant A $( \pm$ SE) & Statistics \\
\hline Coker Un & Coker Spod & $1.216 \pm 0.161$ & $0.108 \pm 0.030$ & $0.76 \pm 0.062$ & $V=209 ; p<0.001$ \\
\hline Coker Un & Coker JA & $0.998 \pm 0.151$ & $0.334 \pm 0.062$ & $0.39 \pm 0.119$ & $V=181 ; p=0.003$ \\
\hline RNAi 1 Un & RNAi 1 Spod & $1.082 \pm 0.137$ & $0.324 \pm 0.077$ & $0.57 \pm 0.079$ & $V=204 ; p=0.001$ \\
\hline RNAi 1 Un & RNAi 1 JA & $1.191 \pm 0.198$ & $0.461 \pm 0.098$ & $0.39 \pm 0.105$ & $V=186 ; p=0.003$ \\
\hline Coker Un & RNAi 1 Un & $0.628 \pm 0.086$ & $0.709 \pm 0.145$ & $0.12 \pm 0.135$ & $V=128 ; p=0.737$ \\
\hline Coker Spod & RNAi 1 Spod & $0.549 \pm 0.156$ & $0.652 \pm 0.106$ & $-0.28 \pm 0.145$ & $V=59 ; p=0.926$ \\
\hline Coker JA & RNAi 1 JA & $0.391 \pm 0.102$ & $0.760 \pm 0.126$ & $-0.30 \pm 0.124$ & $V=43 ; p=0.120$ \\
\hline RNAi 2 Un & RNAi 2 Spod & $1.291 \pm 0.167$ & $0.169 \pm 0.053$ & $0.69 \pm 0.090$ & $V=201 ; p<0.001$ \\
\hline RNAi 2 Un & RNAi 2 JA & $1.017 \pm 0.145$ & $0.291 \pm 0.068$ & $0.52 \pm 0.100$ & $V=195 ; p<0.001$ \\
\hline Coker Un & RNAi 2 Un & $0.706 \pm 0.130$ & $0.836 \pm 0.106$ & $-0.18 \pm 0.096$ & $V=50 ; p=0.120$ \\
\hline Coker Spod & RNAi 2 Spod & $0.415 \pm 0.088$ & $0.612 \pm 0.108$ & $-0.18 \pm 0.129$ & $V=72 ; p=0.461$ \\
\hline Coker JA & RNAi 2 JA & $0.664 \pm 0.123$ & $0.598 \pm 0.095$ & $0.02 \pm 0.104$ & $V=108 ; p=0.926$ \\
\hline
\end{tabular}

reduced $\delta$-cadinene synthease activity that has proven to be completely seed-specific $[9,10,13]$. Also, terpenoid production in the second line (RNAi 2) was similar compared to the conventional control plants and was induced in both lines in response to caterpillar-damage or treatment with jasmonic acid. The differences between the two ULGCS cotton lines might be due to the tissue culture and very long regeneration process resulting in somaclonal variation. Secondary plant metabolite concentrations can vary among different cultivars, varieties or genotypes which is also true for cotton [14]. Therefore, some variation among different lines of ULGCS and conventional cotton can be expected. Such variation, however, should be considered when selecting the most suitable transformation lines for further development. Certain differences in terpenoid concentrations among different RNAi lines were reported before [11]. Our line RNAi 2 has recently been approved by US regulators as event TAM66274 [10].

As expected, feeding bioassays with $S$. littoralis revealed that their performance (weight increase, consumed leaf area) was reduced on induced cotton plants. When plants were undamaged or had been treated with jasmonic acid, no difference in $S$. littoralis performance among the three cotton lines was observed. However, when plants had previously been damaged by caterpillars, $S$. littorals performance was significantly better on the two ULGCS lines as compared to the Coker 312 control plants. The fact that terpenoid concentrations were lower in RNAi 1 plants as compared to RNAi 2 plants, had no effect on the caterpillar performance. The effects might thus be due to some other phytochemical changes in the plant as a response to caterpillar damage [15].

Behavioral choice experiments revealed no preference by $S$. littoralis larvae for any of the three cotton lines. However, leaf discs from uninduced plants were always preferred over those from induced plants.

The picture is clearer for the cotyledons of ULGCS plants as they were significantly more susceptible to $S$. littoralis damage. A very low gossypol content in ULGCS cotyledons has been reported before [11]. Most likely, the cotyledons receive their terpenoids from the seed itself, which would explain why ULGCS lines contain low amounts of terpenoids [11]. Although S. littoralis is not a relevant pest of cotton seedlings, other herbivores, e.g. thrips or flea beetles, can cause significant damage during this stage [16]. Therefore, pest management strategies for ULGCS cotton need to take this potential vulnerability into account to prevent yield losses due to insect damage in this very early growth stage. This might not be a concern in production regions where neonicotinoid seedtreatments for cotton is common $[17,18]$.

Plants can react to herbivore damage by either tolerating the damage or actively defending themselves [19]. Both strategies are costly either due to association costs or biomass loss $[20,21]$, but the costs in both cases are outweighed when reproductive success is maintained [22]. However, under agronomical situations, high yield is economically important rather than successful reproduction. Therefore, plants are often grown under 
quasi pest free conditions, where herbivores are removed for example by using pesticides [1]. In recent years, a goal has been to reduce the application of pesticides by strengthening herbivore resistance traits. In cotton, inducible terpenoids play an important role and plants that do not produce these terpenoids are less resistant against herbivores and pathogens [6, 23], therefore, maintaining this resistance trait is also highly important for the long-term success of ULGCS cotton. Herbivore damage in cotton plants increases the abundance of terpenoids and other relevant resistance traits, which has a negative impact on herbivores [24]. While previous studies have looked into the terpenoid-based resistance mechanisms of ULGCS cotton, they have not addressed the impact of the trait on plant-insect interactions. A previous study tested the disease resistance of ULGCS seedlings and did not find any difference in their susceptibility to the fungus $R$. solani [11]. Our study complements this previous work by expanding resistance research in ULGCS cotton into insect resistance mechanisms. While cotton terpenoids play a role in both herbivore and pathogen resistance, these are thought to be regulated differently and therefore the transformation could affect these pathways differently. Our research indicates that the terpenoid-based insect resistance remains intact in the ULGCS lines tested, at least when true leaves are considered.

As our study was conducted under controlled laboratory conditions it is important to validate the results in the field with varying climate conditions and under the pressure of multiple herbivores belonging to different feeding guilds. A study by Palle et al. [13] found that terpenoid concentrations in ULGCS (including the RNAi 1 line used in the present study) and conventional plants were similar under field conditions. While we found a reduced terpenoid (hemigossypolone, gossypol) concentration in the RNAi 1 line, these differences in the results are possibly explained by different responses of the plants under field and greenhouse conditions. Overall, 7 years of field studies at Texas A\&M have not indicated any difference in pest susceptibility between the RNAitransgenic ULGCS lines and the untransformed plants $[10,13]$. In addition, eight different, multi-state, regulatory field trials conducted over 2 years across the cotton belt in the U.S. did not show a higher degree of pest-susceptibility of the RNAi lines compared to the non-transgenic control [25].

While we could not find a strong impact of ULGCS cotton on the direct, terpenoid-based insect resistance traits, indirect resistance traits were not considered in the present study. The latter are important because they attract/arrest natural enemies of the cotton herbivores to infected plants [19]. A key part in this recruitment is the release of herbivore-induced volatiles (HIVPs). This system is well studied in cotton and the volatile blend of cotton is rich in small terpenes such as $\delta$-cadinene [26]. As ULGCS cotton suppresses the production of $\delta$-cadinene synthase in seeds, this could affect the composition of the plants volatile blend, however, only during the very early stages of the growth.

\section{Conclusions}

We found that the terpenoid-based constitutive and induced insect resistance in ULGCS cotton lines is little affected by the genetic transformation when compared to conventional cotton. At the same time, these plants have a greatly reduced amount of terpenoids in the seeds as intended. As a consequence, gossypol content and thus resistance in cotyledons is weaker and $S$. littoralis larvae perform better on ULGCS cotyledons than on conventional ones. Therefore, ULGCS cotton plants might be more susceptible to insect damage during the early stages after germination and may require additional chemical protection, as it is routinely practiced by farmers in many cotton-growing countries.

\section{Methods}

Insects

Eggs of S. littoralis were provided by Syngenta and sent on a weekly basis from Stein, Switzerland. Larvae were kept at $25^{\circ} \mathrm{C}, 70 \% \mathrm{RH}$, a 16:8 day:night light cycle, and fed with Heliothis Stonefly Diet (Ward Science, Rochester NY, USA) until they reached the desired stage.

\section{Plants}

Plants of two ULGCS lines (RNAi 1: line 66-49B; RNAi 2: line 66-274) were used (see [11] for a description of the lines). Additionally, non-transgenic Coker 312 plants, the paternal (untransformed) cultivar which was used to create the two ULGCSs lines, were serving as a control (termed "conventional cotton"). All plant material has been provided by Keerti S. Rathore (Texas A \& M University, USA). Plants were initially grown in small pots in a climate chamber with $25^{\circ} \mathrm{C}, 70 \% \mathrm{RH}$, and a $16: 8$ day:night light cycle. After 10 days, the plants were transferred to 31 plastic pots, containing humus-rich soil enriched with $15 \mathrm{mg}$ of the slow release fertilizer Manna Cote $4 \mathrm{M}$ (Wilhelm Haug GmbH, Ammerbuch, Germany). They were moved to a climate controlled greenhouse and grown under the same conditions as in the climate chamber. After 4 weeks the plants were once fertilized with $10 \mathrm{~N}: 10 \mathrm{P}: 8 \mathrm{~K}$ at $10 \mathrm{ml} / \mathrm{L}$. In the greenhouse, plants were enclosed in gauze cages (height, $71 \mathrm{~cm}$; diameter, $35 \mathrm{~cm}$; mesh-width, $0.264 \mathrm{~mm}$ ) to protect them from glasshouse pests. Plants needed for experiments at the cotyledon stage were used 10 days after 
sowing, while plants used for the true leave assays were used after $4-5$ weeks.

\section{Plant induction}

Plants that had four fully-developed true leaves and did not show any signs of damage caused by herbivores or diseases were treated in one of three different ways: (i) plants were left untreated (control), (ii) plants were induced with $4.8 \mu \mathrm{mol}$ of jasmonic acid (Sigma-Aldrich; MO, USA), or (iii) plants were induced by releasing three pre-weighted 2nd instar S. littoralis larvae on the second or third true leaf (counted from the bottom of the plant). The larvae were contained on the leaf using an organdy cloth bag. Jasmonic acid was applied in $1 \mathrm{ml}$ of an ethanol: water solution $(20 \mu \mathrm{l}$ ethanol: $980 \mu \mathrm{l}$ of water). The solution was directly applied to the stem of the plant, thereby allowing uptake of jasmonic acid by above- and below-ground tissue. Hagenbucher et al. [27] and Eisenring et al. [28] showed that the application of jasmonic acid induces the terpenoid-based defence in cotton. After 1 week the plants were used for the different experiments. Damage and performance of S. littoralis were assessed by weighing the surviving larvae and measuring the feeding damage by scanning the leaves and measuring the consumed leaf area using the software Image J 1.48 (NIH, USA; [29]).

\section{Performance assay with cotyledons}

This feeding experiment was conducted to assess the performance of $S$. littoralis larvae on cotyledons of the different cotton lines. Plants were grown as described above but remained in the climate chamber. The plants were used 10 days after sowing. All plants remained untreated (no induction). From these cotyledons, $1 \mathrm{~cm}$ leaf discs were taken and transferred to the wells of 128-well plastic bioassay trays which were sealed with 16 cell tray covers (Bio-Serv; Flemington, NJ, USA). The remaining leaf material was stored at $-80^{\circ} \mathrm{C}$ and used for terpenoid quantification. The bottom of each well was covered with a $1 \%$ agar-gel to keep the leaf discs moist. Agar was used since it completely binds water and therefore prevents the formation of small droplets that could be hazardous for the extremely sensitive neonates. On each disc, a single neonate $S$. littoralis was placed. After 4 days the larvae were transferred to a $3 \mathrm{~cm}$ diameter Petri-dish, containing a new $3 \mathrm{~cm}$ leaf disc from the second cotyledon of the same plant. The bottom of the Petri-dish was covered with plaster (Quickmix; QuickmixGruppe, Osnabrück, Germany) to provide moisture. Plaster was used for older larvae as it has the advantage that it cannot be consumed and does not provide energy compared to agar. After a total of 7 days, the assay was terminated. The following data were recorded after four and 7 days: larval survival, weight, larval stage, and consumed leaf area using Image J 1.48. A total of 30-32 larvae was tested per cotton line.

\section{Performance assay with true leaves}

This feeding experiment was conducted to assess if the performance of $S$. littoralis is affected by transformation or induction. Therefore, the youngest fully developed leaf from plants of all three lines and all three induction treatments were collected to be used in a feeding assay.

From these leaves, $1 \mathrm{~cm}$ leaf discs were taken and transferred to the wells of 128-well plastic bioassay trays which were sealed with 16 cell tray covers (Bio-Serv). The remaining leaf material was stored at $-80^{\circ} \mathrm{C}$ and used for terpenoid quantification. The bottom of each well was covered with a $1 \%$ agar-gel to keep the leaf discs moist, as described above. On each leaf disc, a single neonate $S$. littoralis was placed. After 4 days, the larvae were transferred to a $3 \mathrm{~cm}$ diameter Petri-dish, containing a new $3 \mathrm{~cm}$ leaf disc from the same plant, retrieved from the actual youngest fully-developed leaf at that time. The bottom of the Petri-dish was covered with plaster (Quickmix) to provide moisture. After three additional days, the experiment was terminated. The following data were recorded after 4 and 7 days: larval survival, weight, and the leaf area consumed. Each plant used in this experiment was used to feed four different larvae. A total of 20 plants were tested in this way per plant type and treatment. Data for larvae feeding on leaves from one plant were pooled to avoid pseudo-replication, resulting in 20 replications (with a total of 80 larvae tested per treatment). Data recorded for larvae that died during the experiment were not included in the analysis of sublethal parameters.

For a subset of larvae on untreated plants of the three plant lines, the larvae were allowed to complete development to the pupal stage. From each plant, one larva surviving the first 7 days was selected randomly and fed with leaf discs from the same plant until pupation or death. Survival, development time, sex ratio, weight after 14 days and pupal weight (3 days after pupation) were recorded. This part of the experiment was not conducted with induced plants, because high food consumption of late instar S. littoralis makes it difficult to supply the larvae with leaves of consistent quality (degree of induction) over the entire duration of the experiment.

\section{Preference assay}

This experiment was conducted to establish if transformation and terpenoid induction affect the feeding preference of S. littoralis under choice conditions. At the onset of the experiment, the youngest fully developed leaf of each plant was harvested and leaf-discs of $2 \mathrm{~cm}$ 
diameter were cut. Leaf-discs from two different plants were placed on opposite sides of a Petri-dish (diameter $9 \mathrm{~cm}$ ) and a single $3 \mathrm{rd}$ instar $S$. littoralis larva was released in the middle. The Petri-dishes were then moved to a climate cabinet $\left(25^{\circ} \mathrm{C}, 70 \% \mathrm{RH}\right.$, and a $16: 8$ day:night light cycle). After $24 \mathrm{~h}$ the consumed leaf surface was quantified for both leaf discs by scanning the discs and measuring the consumed leaf area using Image J 1.48. From this dataset, a preference index was calculated: (leaf area consumed Plant A - leaf area consumed Plant B)/total leaf area consumed. Discs from one plant-pair were used to test three different larvae, in total 20 plantpairs were tested for each comparison. The leaf material that remained after cutting the three discs was stored at $-80^{\circ} \mathrm{C}$ and used for terpenoid quantification. To avoid pseudo-replications, data for larvae tested with leaf discs from the same plant were pooled, resulting in 20 replications (with a total of 60 larvae tested per treatment).

\section{Terpenoid quantification}

Plant material collected in the different experiments was analyzed via HPLC system (1090 Series 219 II, HewlettPackard, Palo Alto, USA; column: Varian Polaris Amide C-18 column, $150 \times 2.0 \mathrm{~mm}, 3 \mu \mathrm{m}$, equipped with a precolumn C18, 4 × $3.0 \mathrm{~mm}$, Supelco Security Guard System). HPLC analyses followed the methodology described by Hagenbucher et al. [27], which is capable of quantifying gossypol, hemigossypolone, heliocides $\mathrm{H} 1+\mathrm{H} 4$, and heliocides $\mathrm{H} 2+\mathrm{H} 3$.

\section{Statistics}

The cotyledon assay was analyzed using a GLM with plant type as independent variable and the dependent variables survival rate (quasipoisson, due to overdispersion), weight (gamma distribution), and consumed leaf area (gamma distribution). Means were subsequently separated using Tukey's HSD test.

The performance assays with true leaves were analyzed using a GLM with plant type, induction treatment, and the interaction of both as independent variables. Dependent variables were survival rate (quasipoisson, due to overdispersion), weight (gamma distribution), and consumed leaf area (gamma distribution). Means were subsequently separated using Tukey's HSD test. The data for larvae that were fed during their entire development time with the three cotton types was analyzed using a GLM. Therefore, we used plant type as independent variable while pupal weight (gamma distribution), development time (poisson distribution), weight after 14 days (gamma distribution) and sex ratio (binomial distribution) were used as the dependent variables.

To analyze the preference (indicated as consumed leaf area) of $S$. larvae for one of two leaf discs in the choice experiments, one-sided Wilcoxon signed-rank tests were conducted. The preference indices among different choice tests were subsequently compared using twosided Wilcoxon signed-rank tests.

The terpenoid content of mature cotton leaves were analyzed using a GLM with plant type, induction treatment, and the interaction of both as independent variables. Dependent variables were gossypol concentration (normal distribution), hemigossypolone concentration (normal distribution), and Heliocide $1+4$ concentration (normal distribution). For an in depth analyses the concentrations of the terpenoids were analyzed with a GLM (assuming normal distribution) for each of three treatments (untreated, S. littoralis infested, and jasmonic acid-treated). These analyses were done separate for each of the three terpenoid classes. Means were subsequently separated using Tukey's HSD test.

All data were analyzed using R3.1.0 statistical software (https://www.r-project.org/).

\section{Additional file}

\section{Additional file 1: Figure S1. Interaction plot for leaf area consumed by S. littoralis larvae after 4 days. Figure S2. Interaction plot for leaf area consumed by S. littoralis larvae after 7 days. Figure S3. Interaction plot for weight of S. littoralis larvae after 4 days. Figure S4. Interaction plot for weight of S. littoralis larvae after 7 days. (DOCX 3984 kb)}

\section{Abbreviations}

HPLC: High-performance liquid chromatography; RNAi 1: ULGCS cotton line 66-49B; RNAi 2: ULGCS cotton line 66-274; RNAi: RNA interference; ULGCS: Ultra-low gossypol cottonseed

\section{Acknowledgements}

We thank Syngenta (Stein, Switzerland) for providing egg batches of S. littoralis.

\section{Authors contributions}

JR and MM conceived the project. SH, ME, MM, KSR and JR designed the experiments. SH and ME performed the experiments. SH analysed the data and wrote the manuscript. All authors contributed to the writing and approved the manuscript.

\section{Funding}

This research was supported by the Swiss National Science Foundation (SNF grant number 31003A-149794). The funders had no role in study design, data collection and analysis, decision to publish, or preparation of the manuscript.

\section{Availability of data and materials \\ The authors confirm that all data underlying the findings are fully available without restriction. All relevant data are available on the figshare Data Repository (http://dx.doi.org/10.6084/m9.figshare.8329760).}

Ethics approval and consent to participate

Not applicable.

Consent for publication

Not applicable.

\section{Competing interests}

$\mathrm{SH}, \mathrm{ME}, \mathrm{MM}, \mathrm{JR}$ declare that they have no competing interests. KR is inventor on U.S. patents 7999148, 8987554, 10273495 related to ULGCS technology and U.S. patent application no. 16030593 (Publication no. 20190008113 A1) related to TAM66274. 


\section{Author details}

${ }^{1}$ Agroscope, Research Division Agroecology and Environment, Reckenholzstrasse 191, 8046 Zürich, Switzerland. ${ }^{2}$ Department of Soil and Crop Sciences, Institute for Plant Genomics \& Biotechnology, Texas A\&M University, College Station, TX, USA.

Received: 30 November 2018 Accepted: 3 July 2019

Published online: 18 July 2019

\section{References}

1. Trapero C, Wilson IW, Stiller WN, Wilson L. Enhancing integrated pest management in GM cotton systems using host plant resistance. Front Plant Sci. 2016;7:500.

2. Cai Y, Xie Y, Li J. Glandless seed and glanded plant research in cotton. A review. Agron Sustain Dev. 2010;30:181-90.

3. Watkins SE, Waldroup PW. Utilization of high protein cottonseed meal in broiler diets. J Appl Poult Res. 1995;4:310-31.

4. Bertrand JA, Sudduth TQ, Condon A, Jenkins TC, Calhoun MC. Nutrient content of whole cottonseed. J Dairy Sci. 2005;88:1470-7.

5. Risco CA, Chase CC Jr. Gossypol. In: D'Mello JPF, editor. Handbook of plant and fungal toxicants. Boca Raton, FL: CRC Press; 1997. p. 87-98.

6. Hagenbucher S, Olson D, Ruberson J, Wäckers F, Romeis J. Resistance mechanisms against arthropod herbivores in cotton and their interactions with natural enemies. Crit Rev Plant Sci. 2013:32:458-82.

7. Jenkins JN, Maxwell FG, Lafever HN. The comparative preference of insects for glanded and glandless cotton. J Econ Entomol. 1966:59:352-6.

8. Sunilkumar G, Connell JP, Smith CW, Reddy AS, Rathore KS. Isolation and functional characterization of alpha-globulin promoter from cotton in transgenic cotton, Arabidopsis and tobacco. Transgenic Res. 2002;11:347-59.

9. Sunilkumar G, LeAnne M, Campbell L, Puckhaber LS, Stipanovic RD, Rathore KS. Engineering cottonseed for use in human nutrition by tissue-specific reduction of toxic gossypol. Proc Natl Acad Sci U S A. 2006;103:18054-9.

10. USDA-APHIS. Texas A\&M AgriLife Research; Determination of Nonregulated Status of Cotton Genetically Engineered for Ultra-low Gossypol Levels in the Cottonseed. Docket No. APHIS-2017-0097. 2018. https://www.govinfo.gov/ content/pkg/FR-2018-10-17/pdf/2018-22545.pdf . Accessed 28 May 2019.

11. Rathore KS, Sundaram S, Sunilkumar G, Campbell LM, Puckhaber L, Marcel S, Palle SR, Stipanovic RD, Wedegaertner TC. Ultra-low gossypol cottonseed: generational stability of the seed-specific, RNAimediated phenotype and resumption of terpenoid profile following seed germination. Plant Biotech J. 2012:10:174-83.

12. Pogue G. A world revision of the genus Spodoptera. Mem Am Entomol Soc. 2002:43:1-202.

13. Palle SR, Campbell LM, Pandeya D, Puckhaber L, Tollack LK, Marcel S, Sundaram S, Stipanovic RD, Wedegaertner TC, Hinze L, Rathore KS. RNAimediated ultra-low gossypol cottonseed trait: performance of transgenic lines under field conditions. Plant Biotech J. 2013;11:296-304.

14. Altmann DW, Stipanovic RD, Bell AA. Terpenoids in foliar pigment glands of $\mathrm{a}, \mathrm{D}$ and $\mathrm{AD}$ genome cottons: introgression potential for pest resistance. J Hered. 1990;81:447-54.

15. Eisenring M, Glauser G, Meissle M, Romeis J. Differential impact of herbivores from three feeding guilds on systemic secondary metabolite induction, phytohormone levels and plant-mediated herbivore interactions. J Chem Ecol. 2018:44:1178-89.

16. Matthews GA, Tunstall JP. Insect pests of cotton. Wallingford: $C A B$ International; 1994

17. Allen KC, Lutrell RG, Sappington TW, Hesler LS, Papiernik SK. Frequency and abundance of selected early-season insect pests of cotton. J Integr Pest Manage. 2018;9:20

18. North JH, Gore J, Catchot AL, Stewart SD, Lorenz GM, Musser FR, Cook DR, Kerns DL, Dodds DM. Value of neonicotinoid insecticide seed treatments in mid-south cotton (Gossypium hirsutum [Malvales: Malvaceae]) production systems. J Econ Entomol. 2018;111:10-5.

19. Schoonhoven LM, van Loon JJA, Dicke M. Insect-plant biology. 2nd ed. Oxford: Oxford University Press; 2005.

20. Strauss SY, Agrawal AA. The ecology and evolution of plant tolerance to herbivory. Trends Ecol Evol. 1999;14:179-85.

21. Neilson EH, Goodger JQ, Woodrow IE, Møller BL. Plant chemical defense: at what cost? Trends Plant Sci. 2013:18:250-8.

22. Heil M. Ecological costs of induced resistance. Curr Opin Plant Biol. 2002;5:345-50.
23. Bottger GT, Sheehan ET, Lukefahr MJ. Relation of gossypol content of cotton plants to insect resistance. J Econ Entomol. 1964;57:283-5.

24. Zebelo S, Disi J, Balusu R, Reeves B, Fadamiro H. Spodoptera exigua modulates gossypol biosynthesis in cotton Gossypium hirsutum. J Plant Interact. 2017;12:121-7.

25. USDA-APHIS. Petition for Determination of Non-regulated Status for UltraLow Gossypol Cotton seed TAM66274. Texas A\&M AgriLife Research, IPGB2017-001. 2017. https://www.aphis.usda.gov/brs/aphisdocs/17_29201p.pdf . Accessed 28 May 2019.

26. Hagenbucher S, Birgersson G, Chattington S, Anderson P. Domestication influences choice behavior and performance of a generalist herbivore. Perspect Plant Ecol Evol Syst. 2016;23:63-72.

27. Hagenbucher $\mathrm{S}$, Wäckers FL, Wettstein FE, Olson DM, Ruberson JR, Romeis J. Pest trade-offs in technology: reduced damage by caterpillars in Bt cotton benefits aphids. Proc R Soc B. 2013;280:20130042.

28. Eisenring M, Meissle M, Hagenbucher S, Wettstein F, Naranjo SE, Romeis J. Cotton defense induction patterns under spatially, temporally and quantitatively varying herbivory levels. Front Plant Sci. 2017;8:234.

29. Schneider CA, Rasband WS, Eliceiri KW. NIH image to ImageJ: 25 years of image analysis. Nat Methods. 2012;9:671-5.

\section{Publisher's Note}

Springer Nature remains neutral with regard to jurisdictional claims in published maps and institutional affiliations.
Ready to submit your research? Choose BMC and benefit from:

- fast, convenient online submission

- thorough peer review by experienced researchers in your field

- rapid publication on acceptance

- support for research data, including large and complex data types

- gold Open Access which fosters wider collaboration and increased citations

- maximum visibility for your research: over $100 \mathrm{M}$ website views per year

At BMC, research is always in progress.

Learn more biomedcentral.com/submissions 